\title{
Compositional Algorithms for Succinct Safety Games
}

\author{
Romain Brenguier, Guillermo A. Pérez, \\ Jean-François Raskin*, Ocan Sankur* \\ \{rbrengui, gperezme, jraskin, osankur\}@ulb.ac.be \\ Université Libre de Bruxelles - Brussels, Belgium
}

\begin{abstract}
We study the synthesis of circuits for succinct safety specifications given in the AIG format. We show how AIG safety specifications can be decomposed automatically into sub-specifications. Then we propose symbolic compositional algorithms to solve the synthesis problem compositionally starting for the sub-specifications. We have evaluated the compositional algorithms on a set of benchmarks including those proposed for the first synthesis competition organised in 2014 by the Synthesis Workshop affiliated to the CAV conference. We show that a large number of benchmarks can be decomposed automatically and solved more efficiently with the compositional algorithms that we propose in this paper.
\end{abstract}

\section{Introduction}

We study the synthesis of circuits for succinct safety specifications given in the AIG format. An AIG file for synthesis describes a circuit that compactly defines a transition relation between valuations for latches, uncontrollable and controllable input signals. The circuit contains a special latch called the error latch. Initially, all latches are false, and the controller chooses values for the controllable input signals so as to always keep the error latch low (safety objective), no matter how the environment chooses values for the uncontrollable input signals. The AIG format is monolithic in the sense that it is not explicitly structured into subsystems. This is unfortunate as in general, complex systems or specifications are built of smaller sub-parts and taking into account this structure may be a definite advantage.

And-Inverter Graphs (AIG) have been proposed as a way to provide a simple and compact file format for a model checking competition affiliated to CAV 2007 (see http://fmv . jku . at/aiger/FORMAT). This format has been extended to be the input format for the 2014 reactive synthesis competition. Because the synthesis competition uses the AIG format, and this format is monolithic, all the tools that took part in the 2014 reactive synthesis competition solved the synthesis problems monolithically. Nevertheless, the specifications that were proposed during the 2014 synthesis competition are, for a large part of them, generated from higher level descriptions of systems that bear structure. For example, two of the most interesting sets of benchmarks, GenBuf and AMBA, are generated from Reactive(1) specifications (a tractable subset of LTL specifications) [3], or directly from LTL specifications that are conjunctions of smaller LTL sub-formulas.

In this paper, we show that part of the structure lost during the AIG format translation can be recovered and used to solve the synthesis problem compositionally. First, we propose a static analysis of the AIG file that returns, when possible, a decomposition of the circuit into smaller sub-circuits with their own safety specifications. Then we provide three different algorithms that first solve the sub-games

*Authors supported by the ERC inVEST (279499) project.

${ }^{\dagger}$ Author supported by F.R.S.-FNRS fellowship.

Černý, Kuncak, Madhusudan (Eds.): Fourth Workshop on Synthesis (SYNT 2015)

EPTCS 202, 2016, pp. 98-111 doi 10.4204/EPTCS.202.7 (c) R. Brenguier et al.

This work is licensed under the Creative Commons Attribution License. 
corresponding to the sub-circuits and then aggregate, following three different heuristics, the results obtained on the sub-games. Namely, once we have the solution of all the sub-games we aggregate them by (i) taking their intersection - which, we show, over-approximates the actual solution of the general game - and applying the usual fixpoint algorithm to it; (ii) assigning a score to each pair of solutions based on the number of variables shared and the size of the BDDs obtained after their intersection and using said score to aggregate (pair by pair) all the solutions; (iii) trying to refine them using information from a single step of the fixpoint computation on the general game (i.e.projecting the resulting "bad" states onto each sub-game). We have implemented the decomposition, the compositional synthesis algorithms, and evaluated the approach on the 2014 reactive synthesis competition benchmarks as well as on new benchmarks produced from large LTL specifications.

Related Work. In [9, 10], compositional algorithms are proposed for the LTL realizability problem. The LTL formulas considered there are assumed to be conjunctions of smaller LTL formulas, and so the structure of the specification is directly available to them, while in our case it has to be recovered. Also, the main data-structures used there are based on antichains while we use BDDs. In symbolic model checking algorithms, partitioned transition relations [6] are widely used whenever the system is made of several components. Here, the goal is to compute the one-step successor states without explicitly computing the conjunction of the transition relations for each component. The image computation is rather done using quantification scheduling heuristics which tries to apply variable quantification as early as possible inside the conjunction; see e.g. [16]. We also use partitioned transition relations in our algorithms: the next-state function for each latch is stored separately. Unlike forward model checking algorithms, synthesis algorithms proceed backwards, so we can use the composition operation provided by BDD libraries to compute predecessors, and we do not need any early quantification heuristics.

Structure of the paper. In Section 2, we fix notation and recall the definitions needed to present our results. Then, in Section 3, we describe the class of decompositions our algorithms accept as input, we give some examples of how to decompose a succinct safety specification given by an extended AIGER file and outline the algorithm we implemented to get such a decomposition. Our algorithms are described in detail in Section 4 and the results of our tests are presented in Section 5

\section{Preliminaries}

Let $\mathbb{B}=\{0,1\}$. Given a set of variables $A$, a valuation over $A$ is an element of $\mathbb{B}^{A}$, and a set of valuations over $A$ is represented by its characteristic function $f: \mathbb{B}^{A} \rightarrow \mathbb{B}$. We will write $f(A)$ to make the dependency on the variables $A$ explicit. Given two disjoint sets of variables $A, B$, let us write $\mathbb{B}^{A, B}$ for $\mathbb{B}^{A} \times \mathbb{B}^{B}$. Consider variable sets $A \subseteq B$. We define the projection of a valuation $v: \mathbb{B}^{B}$ to $A$ as $v \downarrow_{A}: \mathbb{B}^{A}$, with $v \downarrow_{A}(a)=1$ if, and only if $v(a)=1$. We extend this notation to functions $f: \mathbb{B}^{B} \rightarrow \mathbb{B}$ by $f \downarrow_{A}: \mathbb{B}^{A} \rightarrow \mathbb{B}$, defined as $f \downarrow_{A}(v)$ if, and only if $\exists v^{\prime} \in \mathbb{B}^{B}, f\left(v^{\prime}\right)$, and $v=v^{\prime} \downarrow_{A}$. We define the lifting of a set $f: \mathbb{B}^{A} \rightarrow \mathbb{B}$ in $\mathbb{B}^{B}$ by $f \uparrow_{B}(v)=1$ if, and only if $f\left(v \downarrow_{A}\right)=1$. For a set of variables $A=\left\{a_{1}, a_{2}, \ldots\right\}$, let us write $A^{\prime}=\left\{a_{1}^{\prime}, a_{2}^{\prime}, \ldots\right\}$ the set of primed variables. For $f(A)$, let $f\left(A^{\prime}\right)$ denote the characteristic function $f(A)$ where each variable $a \in A$ has been renamed as its primed copy $a^{\prime} \in A^{\prime}$.

Symbolic Games. We formalize the reactive synthesis problem as a two-player turn-based game with safety objective described symbolically. We consider games defined by sequential synchronous circuits, encoded in the AIGER format. More precisely, a game is a tuple $G=\left\langle L, X_{u}, X_{c},\left(f_{l}\right)_{l \in L}\right.$, err $\rangle$, where: 
1. $X_{u}, X_{c}, L$ are finite disjoint sets of Boolean variables representing uncontrollable inputs, controllable inputs, and latches respectively;

2. for each latch $l \in L, f_{l}: \mathbb{B}^{L} \times \mathbb{B}^{X_{u}} \times \mathbb{B}^{X_{c}} \rightarrow \mathbb{B}$ is the transition function that gives the valuation of $l$ in the next step. In practice these functions will be given by And-Inverter Graphs (see below for a definition).

3. err $\in L$ is a distinguished latch which indicates whether an error has occurred. We will often modify the circuit by replacing $f_{\text {err }}$ by some other Boolean function $e$, which we denote by $G\left[f_{\text {err }} \leftarrow e\right]$.

A state $q$ of game $G$ is a valuation of latches, that is an element of $\mathbb{B}^{L}$. A valuation $v$ in game $G$ is a valuation of latches and inputs, that is an element of $\mathbb{B}^{L, X_{u}, X_{c}}$. We denote the global transition function $\delta: \mathbb{B}^{L} \times \mathbb{B}^{X_{u}} \times \mathbb{B}^{X_{c}} \rightarrow \mathbb{B}^{L}$ such that $\delta(v)(l)=f_{l}(v)$ for each latch $l$. An execution from valuation $v$ of the game $G$ is a sequence of valuations $\left(v_{i}\right)_{i \in \mathbb{N}} \in\left(\mathbb{B}^{L, X_{u}, X_{c}}\right)^{\omega}$ such that $v_{0}=v$ and for all $i$,

$$
v_{i+1} \downarrow_{L}=\delta\left(v_{i} \downarrow_{L}, v_{i} \downarrow_{X_{u}}, v_{i} \downarrow_{X_{c}}\right) .
$$

The execution is safe if, for all $i \geq 0$, we have that $v_{i}(\mathrm{err})=0$.

Note that symbolic games define game arenas of exponential size but we will only work on their symbolic representations.

Controller synthesis. The goal of controller synthesis is to find a strategy to determine the controllable inputs given uncontrollable inputs and the current state (i.e., valuation of the latches) to ensure that the error state is not reachable. A strategy is a function $\lambda: \mathbb{B}^{L, X_{u}} \rightarrow \mathbb{B}^{X_{c}}$. An execution $\left(v_{i}\right)_{i \in \mathbb{N}}$ is compatible with $\lambda$ if for all $i \in \mathbb{N}$,

$$
v_{i} \downarrow_{X_{c}}=\lambda\left(v_{i} \downarrow_{L}, v_{i} \downarrow_{X_{u}}\right) .
$$

A strategy $\lambda$ is winning if all executions that are compatible with $\lambda$ are safe. A valuation $v$ is winning if there exists a strategy $\lambda$ that is winning from $v$. We denote $W\left(L, X_{u}, X_{c}\right)$ the winning valuations of $G$, that is the set of valuations that are winning.

And-Inverter Graphs. An And-Inverter Graph (AIG) is a directed acyclic graph with two-input nodes representing logical conjunction (AND gates), terminal nodes representing inputs, and edges that are possibly inverted to denote logical negation (NOT gate). Formally, an AIG is a tuple $G=\langle V, E, \imath\rangle$ such that $(V, E)$ is a directed graph with every vertex having 0 or 2 outgoing edges, and $\imath: E \rightarrow \mathbb{B}$ labels inverted edges with 1 . We depict edges (not) labelled by $l$ as arrows (not) marked with a dark dot. Figure 1 shows a simple AIG with Boolean variables $x_{1}, x_{2}, x_{3}, x_{4}$. Each node in the AIG defines a Boolean function. For example, $v_{1}$ defines the Boolean function $\varphi_{v_{1}} \equiv x_{1} \wedge \neg \varphi_{v_{2}}$, where $\varphi_{v_{2}}$ is the corresponding formula defined by $v_{2}$, since the edge from $v_{1}$ to $v_{2}$ is marked as inverted.

The AIGER format (http://fmv.jku.at/aiger/FORMAT) was defined as a standard file format to describe sequential synchronous circuits (the logic defined as an AIG), and has been used in model checking and synthesis competitions. In the latter case, the inputs are partitioned into controllable and uncontrollable (http://www . syntcomp.org/wp-content/uploads/2014/02/Format.pdf). This is the format that we will assume as representation of the input game for our algorithms. We call an $A I G$ game, a symbolic game described in the AIGER format. 
Binary Decision Diagrams. Internally, our tool uses binary decision diagrams (BDD) [5] to represent Boolean functions used to represent sets of states or (parts of) transition relations. We use classical operations and notation on BDDs and refer the interested reader to [1] for a gentle introduction to BDDs. Projection and lifting of functions are easily implemented with BDDs: projecting is done by an existential quantification and lifting is a trivial operation because it only extends the domain of the function but its logical representation, i.e. its Boolean formula, stays the same.

In our algorithms, we often use BDD operations which implement heuristics to reduce the size of the given BDD, namely, generalized cofactors [12,15]. A generalized cofactor $\hat{f}(X)$ of $f(X)$ with respect to $g(X)$ yields a BDD that matches $f(X)$ inside $g(X)$, and is defined arbitrarily outside $g(X)$. This degree of freedom outside $g(X)$ allows heuristics to reduce the BDD size. We write $\hat{f}(X)=f(X) \Downarrow g(X)$. Formally, we have that $\hat{f}(X) \wedge g(X)=f(X) \wedge g(X)$ and $\hat{f}$ has at most the size of $f$. BDD libraries implement the operations restrict or constrain (see, e.g. [13]), which are specific generalized cofactors.

Classical Algorithms to Solve Safety Games. We recall the basic fixpoint computation for solving safety games, applied here on symbolic safety games. Let $G=\left\langle L, X_{u}, X_{c},\left(f_{l}\right)_{l \in L}\right.$, err $\rangle$ be a symbolic game. The complement of the set $W\left(L, X_{u}, X_{c}\right) \downarrow_{L}$ can be computed by iterating an uncontrollable predecessors operator. For any set of states $S(L)$, the uncontrollable predecessors of $S$ is defined as

$$
\operatorname{upre}_{G}(S)=\left\{q \in \mathbb{B}^{L} \mid \exists x_{u} \in \mathbb{B}^{X_{u}} . \forall x_{c} \in \mathbb{B}^{X_{c}}: \delta\left(q, x_{u}, x_{c}\right) \in S\right\} ;
$$

the dual controllable predecessors operator is defined as

$$
\operatorname{cpre}_{G}(S)=\left\{q \in \mathbb{B}^{L} \mid \forall x_{u} \in \mathbb{B}^{X_{u}} . \exists x_{c} \in \mathbb{B}^{X_{c}}: \delta\left(q, x_{u}, x_{c}\right) \in S\right\} ;
$$

We denote by upre ${ }_{G}^{*}(S)=\mu X .\left(S \cup\right.$ upre $\left._{G}(X)\right)$, the least fixpoint of the function $F: X \rightarrow S \cup$ upre $_{G}(X)$ in the $\mu$-calculus notation (see [8]). Note that $F$ is defined on the powerset lattice, which is finite. It follows from Tarski-Knaster theorem [14] that, because $F$ is monotonic, the fixpoint exists and can be computed by iterating the application of $F$ starting from any value below it, e.g.the least value of the lattice. Similarly, we denote by $\operatorname{cpre}_{G}^{*}(S)=v X .\left(S \cap \operatorname{cpre}_{G}(X)\right)$, the greatest fixpoint of the function $\left.F: X \rightarrow S \cap \operatorname{cpre}_{G}(X)\right)$. Dually, we have that, because $F$ is monotonic, the fixpoint exists and can be computed by iterating the application of $F$ starting from any value above it, e.g.the greatest value of the lattice. When $G$ is clear from the context, we simply write upre (cpre) instead of upre ${ }_{G}\left(\right.$ cpre $\left._{G}\right)$. The Proposition follows from well-known results about the relationship between safety games and these operators (see, e.g., [2]).

Proposition 1. For any symbolic game $G=\left\langle L, X_{u}, X_{c},\left(f_{l}\right)_{l \in L}\right.$, err $\rangle$, we have

- $\operatorname{cpre}^{*}\left((\operatorname{err} \mapsto 0) \uparrow_{L}\right)=\operatorname{cpre}\left(W\left(L, X_{u}, X_{c}\right) \downarrow_{L}\right)$; dually,

- upre* $\left((\operatorname{err} \mapsto 1) \uparrow_{L}\right)=\neg \operatorname{cpre}\left(W\left(L, X_{u}, X_{c}\right) \downarrow_{L}\right)=\operatorname{upre}\left(\neg W\left(L, X_{u}, X_{c}\right) \downarrow_{L}\right)$.

In the rest of the paper, we assume a black-box procedure solve_vals which, for a given symbolic game, computes the corresponding winning valuations. In practice, solve_vals can be implemented using upre or cpre. Formally,

$$
\text { solve_vals }(G)=\left\{\left(q, x_{u}, x_{c}\right) \in \mathbb{B}^{L, X_{u}, X_{c}} \mid q(\text { err })=0 \wedge \delta\left(q, x_{u}, x_{c}\right) \notin \operatorname{cpre}^{*}\left((\text { err } \mapsto 0) \uparrow_{L}\right)\right\} .
$$

Note that solve_vals gives the set of winning valuations, and not the set of winning states. The interpretation of solve_vals $(G)$ is that it is the maximal permissive strategy: any strategy for the controller that ensures to stay within this set is a winning strategy. We also consider procedure solve_states $(G)=$ $\left\{q \in \mathbb{B}^{L} \mid q \in \operatorname{cpre}^{*}\left((\mathrm{err} \mapsto 0) \uparrow_{L}\right)\right\}$ which returns the set of winning states. 
Optimizations Using Generalized Cofactors. Let us now establish the correctness of two optimizations we use in the sequel.

We first formalize the dependence on latches as follows. The cone of influence (see, e.g., [7]) of $e_{i}$, written cone $\left(e_{i}\right)$, is the set of variables on which $e_{i}$ depends, that is, cone $(\Phi) \subseteq L \cup X_{u} \cup \bar{X}_{c}$ is the minimal set of variables such that if $x \in \operatorname{cone}(\Phi)$ then either $(\exists x: \Phi) \nLeftarrow \Phi$ or $x \in \operatorname{cone}\left(f_{y}\right)$ for some $y \in \operatorname{cone}(\Phi) \cap L$. For convenience, we denote by cone $L(\Phi)$ the set cone $(\Phi) \cap L$.

Observe that we have defined the cone of influence of a Boolean function semantically. That is to say, a variable $x$ is in the cone of influence of a function $\Phi$ if and only if the set of valuations satisfying $\Phi$ changes for some fixed valuation of $x$. Since we consider functions given by AIGs, the cone of influence can be over-approximated by exploring the AIG starting from the vertex corresponding to function $\Phi$, adding all latches and inputs visited and the cones of influence of the latches - computed recursively. In our implementation we use this over-approximation when working on the AIG only and we use the definition on the semantics to obtain an algorithm on BDDs - which we use when working with BDDs.

Given an over-approximation $\Lambda$ of the winning valuations $(i)$ we first simplify the transition relation and keep it precise only in $\Lambda$, $(i i)$ we further modify the transition relation by making every transition not allowed by $\Lambda$ go to an error state, i.e.change $f_{\text {err }}$. In fact, correctness of the first optimization requires that the second one be used as well. The following result summarizes the properties of these optimizations.

Lemma 1. For any symbolic game $G=\left\langle L, X_{u}, X_{c},\left(f_{l}\right)_{l \in L}\right.$, err $\rangle$, and any $\Lambda\left(L, X_{u}, X_{c}\right) \supseteq W\left(L, X_{u}, X_{c}\right)$, if we write $f_{l}^{\prime}=f_{l} \Downarrow \Lambda$ for all $l \in L$, we have

$$
\operatorname{solve} \_v a l s(G)=\operatorname{solve}_{-} v a l s\left(\left\langle\operatorname{cone}_{L}(\Lambda), X_{u}, X_{c},\left(f_{l}^{\prime}\right)_{l \in \operatorname{cone}_{L}(\Lambda)}\right\rangle\left[f_{\text {err }}^{\prime} \leftarrow \neg \Lambda\right]\right) \uparrow_{L} .
$$

Proof. We first show that solving the game with error function $\neg \Lambda$ yields the same winning valuations as for $f_{\text {err }}$. For that we will use two basic properties of the winning valuations: first if $f \subseteq f^{\prime}$ then

$$
\text { solve_vals }\left(G\left[f_{\text {err }} \leftarrow f^{\prime}\right]\right) \subseteq \text { solve_vals }\left(G\left[f_{\text {err }} \leftarrow f\right]\right) \text {; }
$$

secondly

$$
\text { solve_vals }\left(G\left[f_{\text {err }} \leftarrow \neg \text { solve_vals }(G)\right]\right)=\operatorname{solve\_ vals}(G),
$$

this is because if an execution compatible with strategy $\lambda$ reaches $\neg$ solve_vals $(G)$, then by definition of winning valuations it can be extended from there to an execution compatible with $\lambda$ that is unsafe. Together with the fact that $f_{\text {err }} \subseteq \neg \Lambda \subseteq \neg W$, these properties imply that solve_vals $(G)=$ solve_vals $\left(G\left[f_{\text {err }} \leftarrow \neg \Lambda\right]\right)$. It is clear that one can consider only the variables in $\operatorname{cone}_{L}(\Lambda)$ for this computation, and thus considering $H=\left(\left\langle\operatorname{cone}_{L}(\Lambda), X_{u}, X_{c},\left(f_{l}\right)_{l \in \operatorname{cone}_{L}(\Lambda)}\right\rangle\left[f_{\text {err }} \leftarrow \neg \Lambda\right]\right)$, we have

$$
\text { solve_vals }(G)=\text { solve_vals }(H) \uparrow_{L} \text {. }
$$

It remains to show that the same set solve_vals $(H)$ is obtained when the functions $f_{l}^{\prime}$ are used transition functions $f_{l}$. Let us denote $G^{\prime}=\left(\left\langle\operatorname{cone}_{L}(\Lambda), X_{u}, X_{c},\left(f_{l}^{\prime}\right)_{l \in \text { cone }_{L}(\Lambda)}\right\rangle\left[f_{\mathrm{err}}^{\prime} \leftarrow \neg \Lambda\right]\right)$. We note that, for any $u \supseteq \neg \Lambda$, the following holds:

$$
\operatorname{upre}_{G}^{\prime}(u) \cup u=\operatorname{upre}_{H}(u) \cup u \text {. }
$$

Hence, it is straightforward to show by induction that solve_vals $(H)=\operatorname{solve}_{-} \operatorname{vals}\left(G^{\prime}\right)$. 


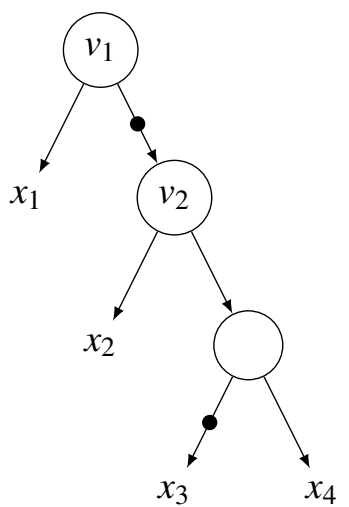

Figure 1: Example AIG

\section{Decomposing the Specification}

In this section, we describe how we decompose the error function $f_{\text {err }}$ of a given symbolic game into a disjunction i.e. $f_{\text {err }} \equiv\left(\bigvee_{1 \leq i \leq n} e_{i}\right)$. Notice that if a strategy $\lambda\left(L, X_{u}, X_{c}\right)$ ensures that $f_{\text {err }}$ is never true then it also ensures that $e_{i}$ is never true. We will then give algorithms that solve the game where each $e_{i}$ is seen as the error function, and combine the obtained solutions into a global solution.

The rationale behind this approach is that the functions $e_{i}$ do not depend on all latches in general, so solving the game for $e_{i}$ is often efficient.

Sub-game. Given a decomposition of $f_{\text {err }}$, we define a sub-game $G_{i}$ by replacing the error function by $e_{i}$ and considering only variables in its cone of influence. Formally, we write

$$
G_{i}=\left\langle\operatorname{cone}_{L}\left(e_{i}\right), X_{u}, X_{c},\left(f_{l}\right)_{l \in \operatorname{cone}_{L}\left(e_{i}\right)}\right\rangle\left[f_{\text {err }} \leftarrow e_{i}\right] .
$$

We will often use the notation $G\left[f_{\text {err }} \leftarrow e_{i}\right]$, which consists in replacing the function $f_{\text {err }}$ by $e_{i}$. In practice, the size of the symbolic representation of the sub-games are often significantly smaller than that of the original game. Recall also that winning all the sub-games is necessary to win the global game. We write $W_{i}\left(\right.$ cone $\left._{L}\left(e_{i}\right), X_{u}, X_{c}\right)$ for the winning valuations of $G_{i}$. In the implementation, $S_{i}$ and $S_{i} \uparrow_{L}$ are represented by the same BDD.

Example 1. Consider the AIG shown in Figure 1 where $x_{1}, x_{2}, x_{3}, x_{4}$ are all input variables. We would like to decompose the function defined by the sub-tree rooted at $v_{1}$ (i.e. the whole tree) which we will denote by $\varphi_{v_{1}}$. It should be clear that $\varphi_{v_{1}} \equiv x_{1} \wedge \neg \varphi_{v_{2}}$ where $\varphi_{v_{2}}$ is the function defined by the sub-tree rooted at $v_{2}$. In turn, we also have that $\varphi_{v_{2}} \equiv x_{2} \wedge \neg x_{3} \wedge x_{4}$. If we distribute the disjunction from $\neg f_{v_{2}}$ we get that $\varphi_{v_{1}} \equiv\left(x_{1} \wedge \neg x_{2}\right) \vee\left(x_{1} \wedge x_{3}\right) \vee\left(x_{1} \wedge \neg x_{4}\right)$. Thus, one possible decomposition of $\varphi_{v_{1}}$ would be to take $e_{1}=x \wedge \neg x_{2}, e_{2}=x_{1} \wedge x_{3}$, and $e_{3}=x_{1} \wedge \neg x_{4}$.

The general steps followed in Example 1 above can be generalized into an algorithm which outputs a decomposition of the error function whenever one exists. Intuitively, the algorithm consists in exploring all non-inverted edges of the AIG graph from the vertex which defines the error function. If there are no inverted edges which stopped the exploration, or if all of them lead to leaves, the error function is in fact a conjunction of Boolean variables and can clearly not be decomposed. Otherwise, there is at least one inverted edge leading to a node representing an AND gate. In this case, we can push the 
negation one level down and obtain a disjunction which can be distributed to obtain our decomposition. Algorithm 2 details the procedure we have implemented. It takes as input an AIG, whether the error function is inverted, and the vertex $v_{\text {err }}$ which defines the error function. It outputs a set of functions whose conjunction is logically equivalent to the error function.

We have kept our description of Algorithm 2 and Algorithm 1 (called by the former) informal.
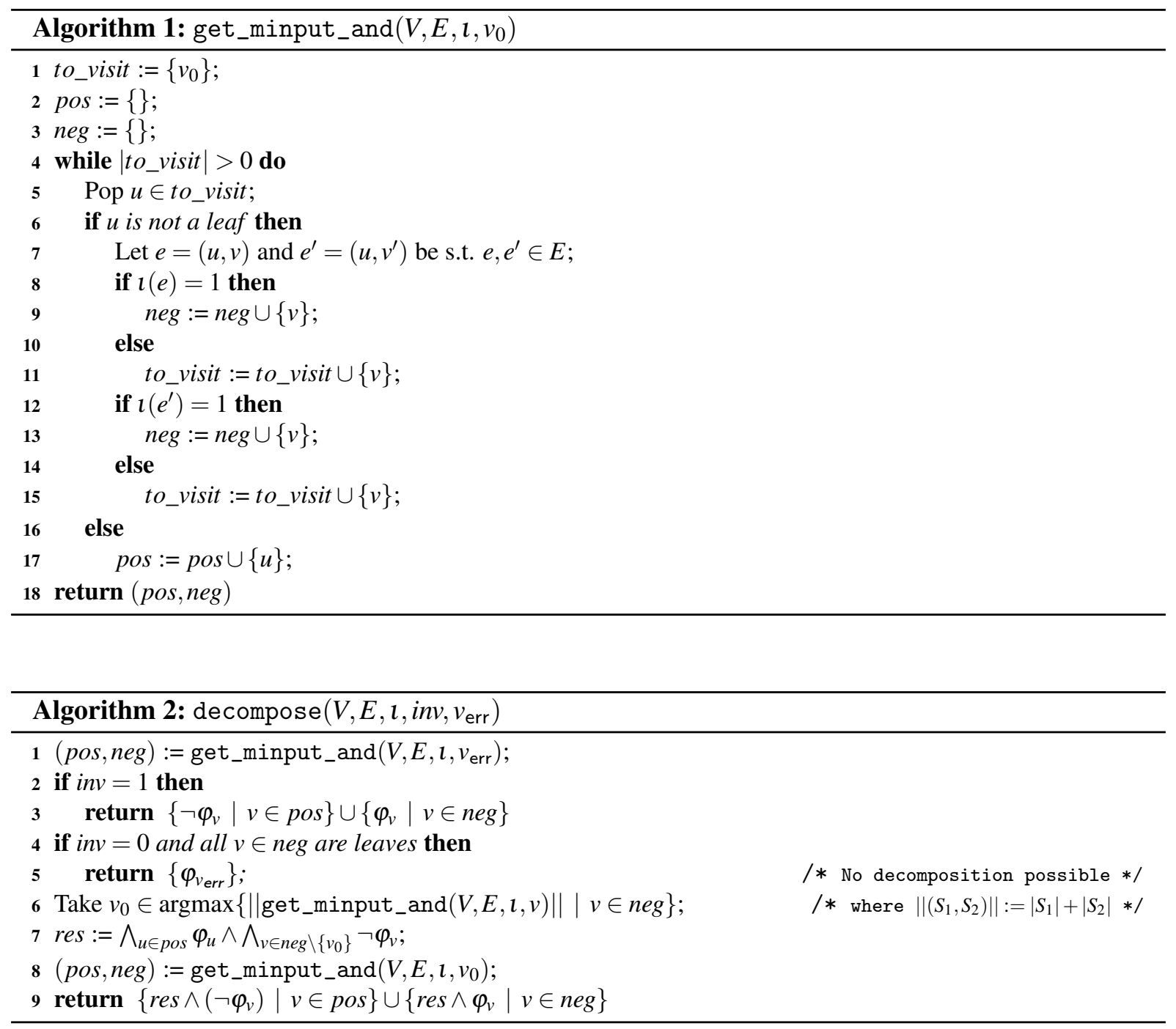

Example 2. Consider a formula given by a set of assumption formulas $\left\{A_{i}\left(L, X_{u}\right) \mid 1 \leq i \leq n\right\}$ and a set of guarantees $\left.\left\{G_{j}\left(L, X_{u}, X_{c}\right) \mid 1 \leq j \leq m\right\}\right|^{1}$ The system we want to synthesize is expected to determine the controllable inputs in way such that if the assumptions are true, then the guarantees are met. This is formally stated as Equation 1 .

$$
\Phi=\left(\bigwedge_{1 \leq i \leq n} A_{i}\right) \Longrightarrow\left(\bigwedge_{1 \leq j \leq m} G_{j}\right)
$$

\footnotetext{
${ }^{1}$ This is actually the way in which the error formula is stated for, e.g., the AMBA benchmarks.
} 


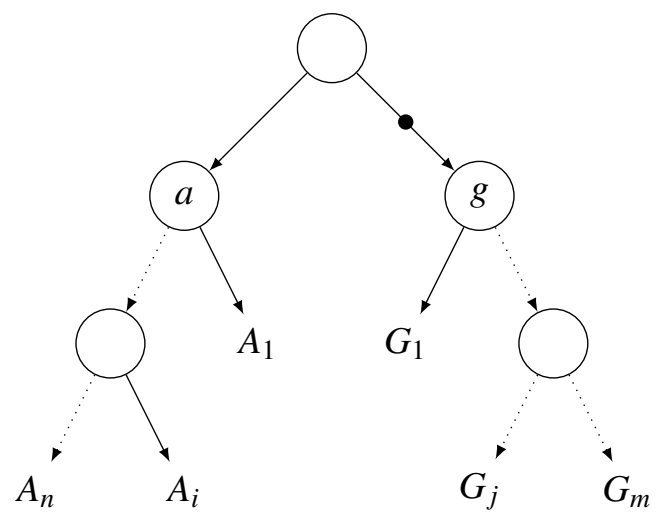

Figure 2: One possible AIG for Equation 1

A natural decomposition for the error function $\neg \Phi$ would be the following: $\bigvee_{1 \leq j \leq m}\left(\neg G_{j} \wedge \bigwedge_{1 \leq i \leq n} A_{i}\right)$. If $\neg \Phi$ were given as the AIG depicted in Figure 2, then it is not hard too see that Algorithm 2 would yield a very similar decomposition. Indeed, as we have not assumed anything in particular about the formulas $A_{i}$ and $G_{j}$ we cannot tell whether Algorithm 1 will explore beyond each $G_{j}$, thus giving us more sub-games than the proposed decomposition. However, in practice, this is even better as smaller sub-games usually depend on less variables. This, in turn, could lead to them being easier to solve.

Lemma 2. For each sub-game $G_{i}$ with new error function $e_{i}$, we have that

$$
W\left(L, X_{u}, X_{c}\right) \subseteq\left(W_{i} \uparrow_{L}\right)\left(L, X_{u}, X_{c}\right)
$$

Proof. For each valuation $v^{\prime} \in W\left(L, X_{u}, X_{c}\right) \downarrow_{\text {cone }_{L}\left(e_{i}\right) \cup X_{u} \cup X_{c}}$, we select a valuation $v \in W\left(L, X_{u}, X_{c}\right)$. Let $\lambda_{v}$ be a winning strategy in $G$ from $v$. Since there is no losing outcome for $\lambda_{v}$, for all $x_{u} \in \mathbb{B}^{X_{u}}$, $\lambda_{v}\left(\delta(v), x_{u}\right)$ is such that $\left(\delta(v), x_{u}, \lambda_{v}\left(\delta(v), x_{u}\right)\right) \in W\left(L, X_{u}, X_{c}\right)$. For all $x_{u} \in \mathbb{B}^{X_{u}}$, we fix $\lambda^{\prime}\left(\delta\left(v^{\prime}\right), x_{u}\right)$ to be $\lambda_{v}\left(\delta(v), x_{u}\right)$. We have that $\left(\delta\left(v^{\prime}\right), x_{u}, \lambda^{\prime}\left(\delta\left(v^{\prime}\right), x_{u}\right)\right) \in W\left(L, X_{u}, X_{c}\right) \downarrow_{\text {cone }_{L}\left(e_{i}\right) \cup X_{u} \cup X_{c}}$ because the transition relations of $G$ and $G_{i}$ coincide on cone $e_{L}\left(e_{i}\right) \cup X_{u} \cup X_{c}$. The strategy $\lambda^{\prime}$ ensures that any execution

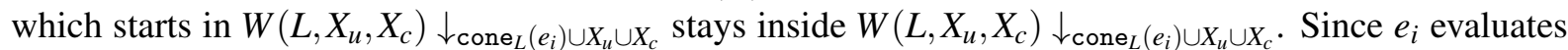
to false on $W\left(L, X_{u}, X_{c}\right)$, these states are not error states in $G_{i}$. Therefore $\lambda^{\prime}$ is winning for all states in $W\left(L, X_{u}, X_{c}\right) \downarrow_{\text {cone }_{L}\left(e_{i}\right) \cup X_{u} \cup X_{c}}$. This implies that $W_{i}$ contains the projection of all winning states of $G$ and therefore $W \subseteq W_{i} \uparrow_{L}$.

\section{Compositional Algorithms}

In this section, we give three algorithms to solve AIG games compositionally. Each algorithm first solves the sub-games, and then combines the solutions using different heuristics. We denote by decompose the procedure that implements the decomposition of $f_{\text {err }}$ described in Section 3 , and returns the set of error functions $e_{i}$. In all three algorithms, we start by solving each sub-game and obtaining the winning valuations $W_{i}\left(L, X_{u}, X_{c}\right)$, for $1 \leq i \leq n$. These steps are given in lines $1-3$, and are common to 11 our algorithms; we assume that solve_vals raises an exception and terminates the program if the sub-game cannot be won. Otherwise, we aggregate the results and solve the global game; for the latter, we adopt a different approach in each of the three algorithms. 


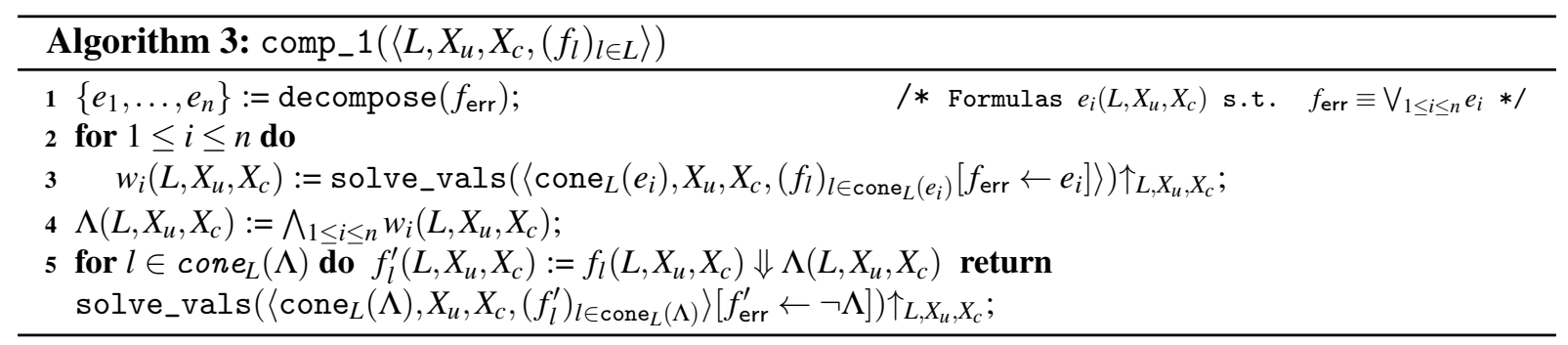

\subsection{Global aggregation}

In Algorithm 3 , we start by computing the intersection of the winning valuations: $\Lambda=\Lambda_{1 \leq i \leq n} W_{i}$. In fact, any valuation that is not in $\Lambda$ is losing in one of the sub-games; thus in the global game. Conversely, a strategy that stays in $\Lambda$ is winning for each sub-game. Therefore, we solve the global game with the new safety objective of avoiding $\neg \Lambda$. Before solving the global game, the algorithm attempts to reduce the size of the transition relations by virtue of Lemma 1 .

Theorem 1. Algorithm 3 computes the winning valuations of the given AIG game.

Proof. We prove first that $W \subseteq \Lambda$ (that is for all valuation $v, W(v) \Rightarrow \Lambda(v)$ ). Since $\neg e_{i} \supseteq W\left(L, X_{u}, X_{c}\right)$, we get - by Lem. 1 - that each $w_{i}\left(L, X_{u}, X_{c}\right)$ is $W_{i} \uparrow_{L}$ where $W_{i}$ is the winning valuations of the sub-game $G_{i}$. If $q \notin \Lambda\left(L, X_{u}, X_{c}\right)$, there is a sub-game $G_{i}$ such that $\pi_{i}(q)$ is not winning. By Lem. 2 , this implies that $q$ is not winning in $G$, hence $q \notin W\left(L, X_{u}, X_{c}\right)$.

From Lem. 1 it then follows that solve_vals $(G)=\operatorname{solve}_{-} \operatorname{vals}\left(G^{\prime}\right) \uparrow_{L}$ and therefore the algorithm computes the correct result.

\subsection{Incremental aggregation}

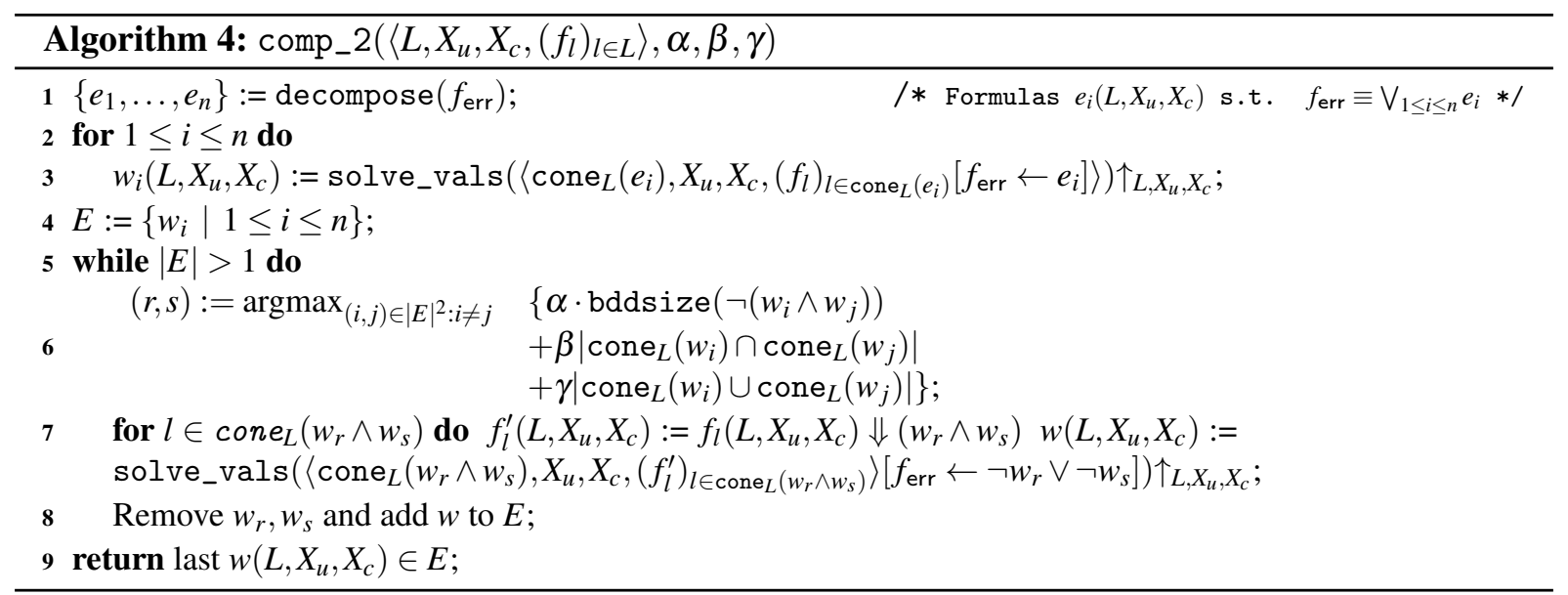

In Algorithm 4, we aggregate the results of the sub-games incrementally: given the list of winning valuations $w_{i}$ for the sub-games, at each iteration, we choose and remove two sub-games $i$ and $j$, solve their conjunction (as in Algorithm 3 , with error function $\neg\left(w_{i} \wedge w_{j}\right)$ ), and add the newly obtained winning valuations back in the list. To choose the sub-games, we use the following heuristics; we assign a score to each pair of sub-games based on the size of the BDD of the error function $\neg\left(w_{i} \wedge w_{j}\right)$, and on the number 
of shared latches, and the number of the latches that appear in either of the sub-games. Intuitively, we prefer to work with small BDDs, and to merge sub-games that share a lot of latches, while yielding a small number of total latches. We thus use a linear combination at line 6 to choose the best scoring pair. In our experiments, we used $\alpha=-2, \beta=1, \gamma=-1$.

Theorem 2. Algorithm 4 computes the winning valuations of the given AIG game.

Proof. Let us denote by $w_{1}^{i}, \ldots, w_{n_{i}}^{i}$ the content of $E$ at the beginning of iteration $i$. We define a function $F$ from winning valuations $w_{j}^{i}$ to subsets of $\{1, \ldots, n\}$. Intuitively, $F\left(w_{j}^{i}\right)$ is the set of sub-games that were solved to obtain $w_{j}^{i}$. For instance, at the first iteration, if sub-games $r, s$ are combined - and the result, $w$, is added to $E-$ then we get $F(w)=\{r, s\}$. For convenience, we assume that $w$ is appended at the end of the sequence $w_{1}^{i}, \ldots, w_{n_{i}}^{i}$ at line 8 .

We proceed by induction on $i$ to define $F$. Initially $F\left(w_{i}^{1}\right)=\{i\}$ for all $1 \leq i \leq n$. For $i>1$, for all $j \neq r, s$, the element $w_{j}^{i}$ remains in the list so $F$ is already defined on $w_{j}^{i}$. For the newly element $w_{n_{i}}^{i}$ we let $F\left(w_{n_{i}}^{i}\right)=F\left(w_{r}^{i-1}\right) \cup F\left(w_{s}^{i-1}\right)$.

We claim that at any iteration $i, w_{j}^{i}$ is the winning valuations of the game whose error function is the disjunction of the negation of the winning valuations of the sub-games in $F\left(w_{j}^{i}\right)$. More precisely,

$$
w_{j}^{i}=\text { solve_vals }\left(\left\langle L, X_{u}, X_{c},\left(f_{l}\right)_{l \in L}\right\rangle\left[f_{\text {err }} \leftarrow \bigvee_{k \in F\left(w_{j}^{i}\right)} e_{k}\right]\right)
$$

The correctness of the algorithm will follow since the sets $F(\cdot)$ are merged at each iteration, and the algorithm always stops with $|E|=1$ and $F(w)=\{1, \ldots, n\}$.

The condition holds initially as shown in Theorem 1. Let $i>1$. As shown in Lem. 1, the generalized cofactor operation applied before the call to solve does not affect the returned set. Let us denote $E_{r}=$ $\bigvee_{k \in F\left(w_{r}^{i-1}\right)} e_{k}$ and $E_{s}=\bigvee_{k \in F\left(w_{s}^{i-1}\right)} e_{s}$. Let us write $\mathscr{E}=E_{r} \vee E_{s}$. We have $E_{r} \Rightarrow \neg w_{r}$ by induction, and similarly $E_{s} \Rightarrow \neg w_{s}$; thus $\mathscr{E} \Rightarrow \neg w_{r} \vee \neg w_{s}$. Moreover, for any $q\left(L, X_{u}\right)$ if the controller plays strategy $x_{u} \in$ $\mathbb{B}^{X_{c}}$ with $\neg w_{r}\left(q, x_{u}\right)$, or $\neg w_{s}\left(q, x_{u}\right)$, then he loses for the error function defined by $\mathscr{E}$. In other terms, $\neg w_{r} \vee$ $\neg w_{s}$ is a subset of losing valuations for error function $\mathscr{E}$, and contains $\mathscr{E}$, the set of states losing in one step. It follows that $w\left(L, X_{u}, X_{c}\right)$ computed at step 7 is the winning valuations for the error function $\mathscr{E}$.

\subsection{Back-and-forth}

In Algorithm 5, we interleave the analysis of the global game (with objective $\Lambda$ ) and the analysis of the sub-games. At each iteration, we extend the losing states $u(L)$ by one step, by applying once the upre operator. We then consider each sub-game, and check whether the new set $u^{\prime}(L)$ of losing states (projected on the sub-game), changes the local winning states. Here, $p_{i}(L)$ is this projection on the local state-space of sub-game $i$. We update the strategies $\lambda_{i}$ of the sub-games when necessary, and restart until stabilization. Because analyzing the sub-games is often more efficient than analyzing the global game, this algorithm improves over Algorithm 3 in some cases (see the experiments' section). A similar idea was used in [10] for the problem of synthesis from LTL specifications.

Theorem 3. Algorithm 5 computes the winning valuations of the given AIG game.

Proof. Let $W(L)$ denote the set of winning states of the game $G$. We consider the following invariant.

$$
\begin{aligned}
& \forall i \in\{1, \ldots, n\}, W(L) \subseteq s_{i}(L), \\
& \operatorname{err} \subseteq u^{\prime}(L) \subseteq \neg W(L) .
\end{aligned}
$$




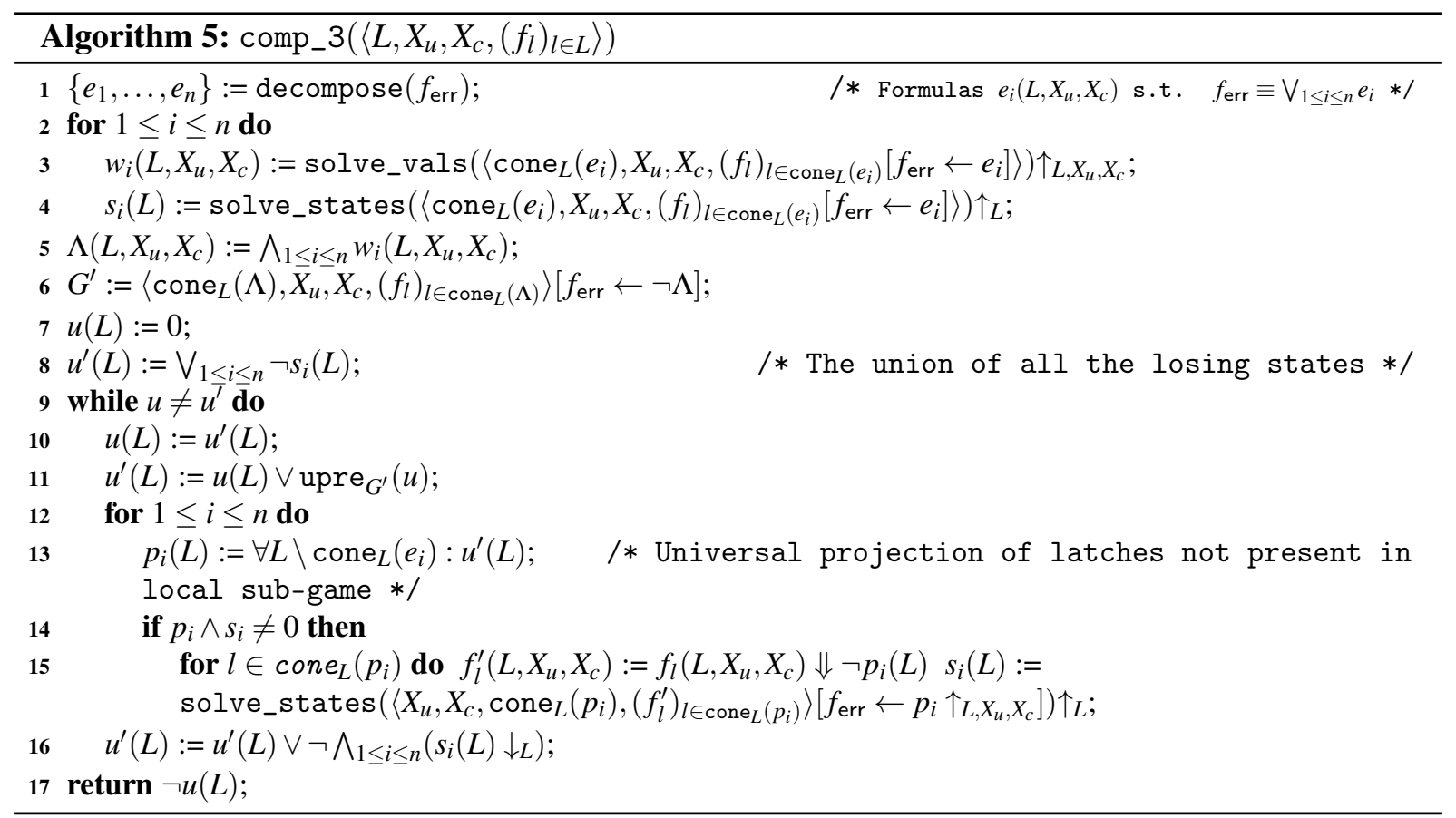

In words, in every iteration, $u^{\prime}(L)$ is contained in the losing valuations of the global game, and each $s_{i}(L)$ contains the winning valuations of $G_{i}$.

Initially, by Algorithm 3, $W \subseteq s_{i}(L)$ for all $i$, and we have err $\subseteq \neg s_{i}(L)$. So err $\subseteq \neg \wedge_{i} s_{i}(L)$. Thus, err $\subseteq u^{\prime}(L)$. Moreover, since $\vee_{i} \neg s_{i}(L) \subseteq \neg W$, we have that $u^{\prime}(L) \subseteq \neg W$.

Consider now iteration $i>1$, and assume the invariant holds at the beginning. $u^{\prime}(L)$ is updated at line 11 . The property err $\subseteq u^{\prime}(L) \subseteq \neg W$ still holds by the definition of the upre operation, and by the fact that the set $u^{\prime}$ can only grow at this step (because of the union).

We consider now the for loop, and show that $W \subseteq s_{i}$ after each iteration. Assume $p_{i} \cap s_{i} \neq \emptyset$ since otherwise $s_{i}$ is not modified. By definition $p_{i} \subseteq u^{\prime}$ thus $p_{i} \subseteq \neg W$. Then the solve function computes the set of states from which the controller can avoid err $\vee p_{i}$. Since err $\vee p_{i} \subseteq \neg W$, we get that $s_{i} \subseteq W$. It follows that $\neg \bigwedge_{i=1}^{n} s_{i} \subseteq \neg W$. Thus, at the last line of the while loop, we have err $\subseteq u^{\prime}(L) \subseteq \neg W$.

Now, line 11 ensures that after iteration $i, u^{\prime}(L)$ contains the $i$-th iteration of the upre fixpoint computation. Hence, the test $u \neq u^{\prime}$ of the while loop ensures that the while loop terminates with $u(L)$ being equal to upre* $(G)$.

\section{Experiments}

We implemented our algorithms in the synthesis tool AbsSynthe [4]. We compare their running times against the most efficient algorithm of AbsSynthe that implements a backward fixpoint algorithm $]^{2}$ This algorithm was the winner of the 2014 Synthesis Competition synthesis track, and the winner of the realizability track at the same competition implemented a similar backward algorithm.

Let us first illustrate the advantage of the compositional approach with two examples. In the first

${ }^{2}$ The new version of AbsSynthe with the implementation of the compositional algorithms can be fetched from https: //github.com/gaperez64/abssynthe. 
set of benchmarks we consider, the controller is to compute the multiplication of two Boolean matrices given as (uncontrollable) input. Since each cell of the resulting matrix depends only on a subset of inputs, namely, on one row and one column, these benchmarks are well adapted for compositional algorithms. Figure 3 compares the performances of the classical algorithm with Algorithm 3 . The classical algorithm was able to solve 36 instances, while the compositional algorithm solved all 75 instances and was significantly faster. The $\mathrm{x}$-axis shows the number of solved benchmarks within the running time given by the y-axis. The second set of benchmarks we consider consist in a washing system made of $n$ tanks. An uncontrollable input can request at any time the tank to be activated, at which point the controller should fill the tank with water, and empty it after at least $k$ steps. Moreover, some subsets of tanks cannot be filled at the same time, and a light is to be on if at least one tank is filled with water. Note that the control strategy for each tank is not independent due to mutual exclusion constraints, and to the light indicator. Algorithm 3 was also more efficient on these benchmarks, as shown on Fig. 4 . The classical algorithm solved 132 benchmarks out of 256, while Alg. 3 solved 152.

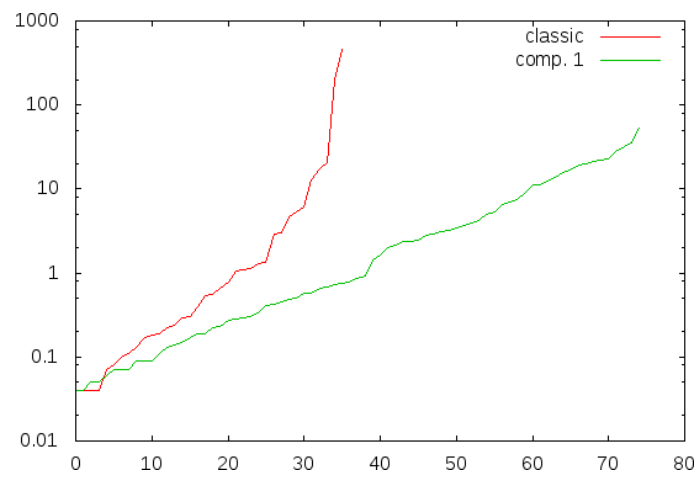

Figure 3: Performances for 75 Boolean matrix multiplication benchmarks for Algorithm 3 and the classical algorithm.

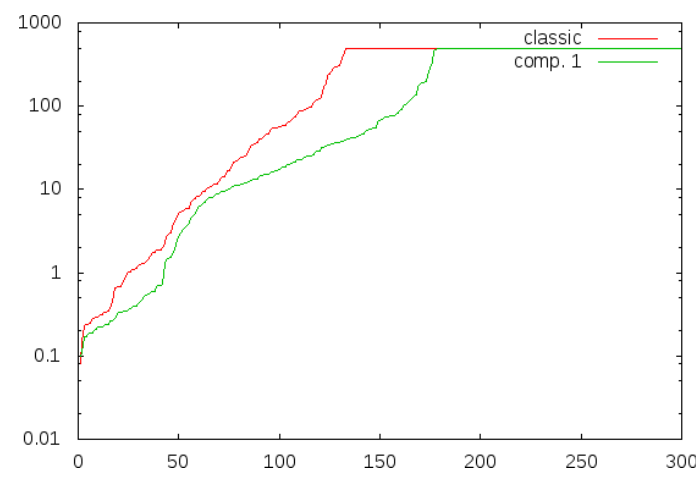

Figure 4: Peformances for the 256 washing system benchmarks for Algorithm 3 and the classical algorithm.

We now evaluate all three compositional algorithms and compare them with the classical algorithm on a large benchmark set of 674 benchmarks. 562 of these benchmarks were provided for the 2014 Synthesis Competition and 105 have been generated by the new version of LTL2AIG [11] which translates conjunctions of LTL specifications into AIG ${ }^{3}$ Among those benchmarks, 351 are decomposable by our static analysis into at least 2 smaller sub-games. More specifically, the average number of sub-games our decomposition algorithm outputs is 29 ; the median is 21 .

In general, the performances of the three compositional algorithms can differ, but they are complementary. Figures 5 to 8 show the performances of the algorithms on several sets of benchmarks. All benchmarks in Figures 5 and 6 are decomposable. Figure 7 shows all the benchmarks we used and Figure 8 shows only those benchmarks from last year's synthesis competition which were based on specifications of the AMBA arbiter.

Conclusion. Even if AIG synthesis problems are monolithic, the experiments show that our compositional approach was able to solve problems that can not be handled by the monolithic backward algorithm; our compositional algorithms are sometimes much more efficient. There are also examples that

\footnotetext{
${ }^{3}$ A collection of benchmarks, including the ones mentioned here, can be fetched from https://github.com/gaperez64/ bench-syntcomp14 and https://github.com/gaperez64/bench-ulb-syntcomp15
} 
can be decomposed but which are not solved more efficiently by the compositional algorithms. So, it is often a good idea to apply all the algorithms in parallel. This portfolio approach improved the performance and was able to solve 20 benchmarks that could not be solved by the fastest algorithms of last year's reactive synthesis competition.

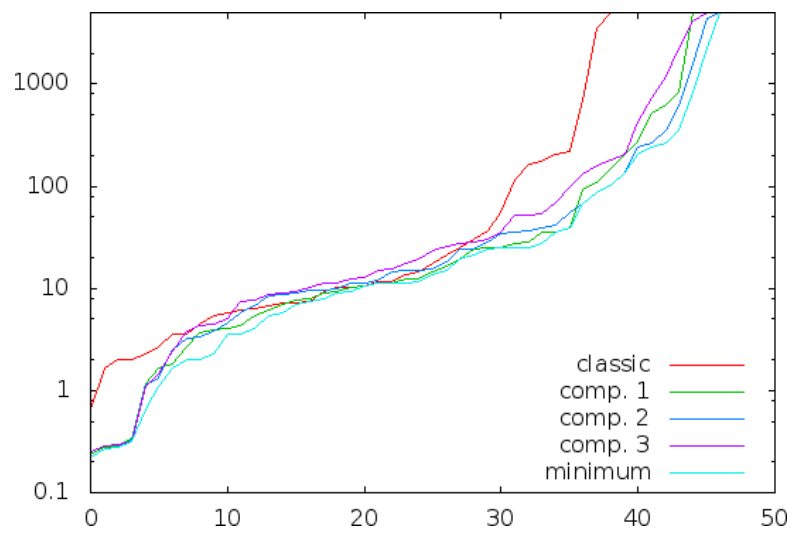

Figure 5: Performances for 68 load-balancing benchmarks translated from LTL. The classical algorithm solves 38 benchmarks, comp. 144 , comp. 2 45 , comp. 345 . In total there are 46 benchmarks that can be solved. The largest example that can be solved has 4005 latches and the smallest example that cannot be solved has 670 latches.

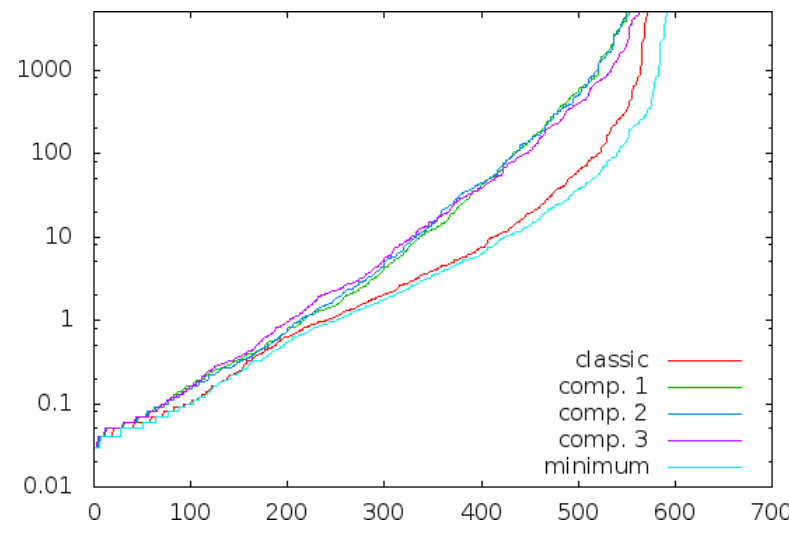

Figure 7: Performances for the 674 benchmarks. The classical algorithm was able to solve 572 benchmarks. 20 more benchmarks were solved by one of the three compositional algorithms.

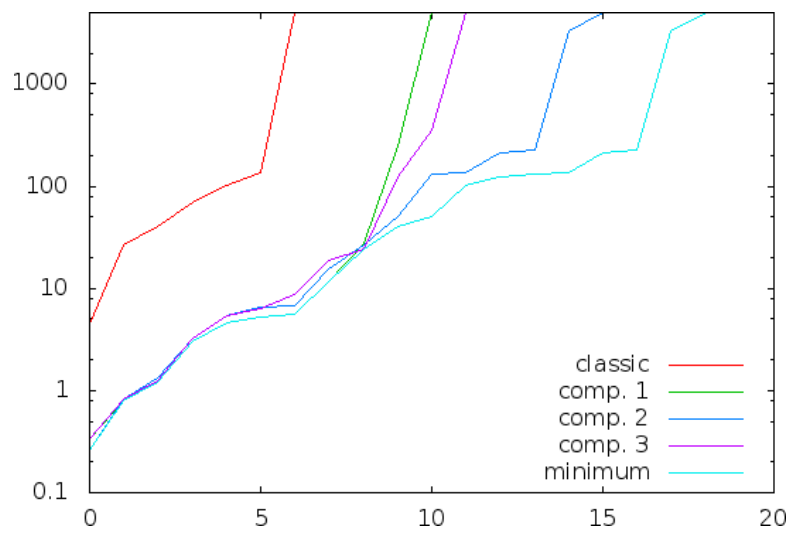

Figure 6: Performances for 46 generalized buffer benchmarks translated from LTL. The classical algorithm solves 6 benchmarks, comp. 1 10, comp. 2 15, comp. 3 11. In total there are 18 benchmarks that can be solved. The largest example that can be solved has 22662 latches and the smallest example that cannot be solved has 590 latches.

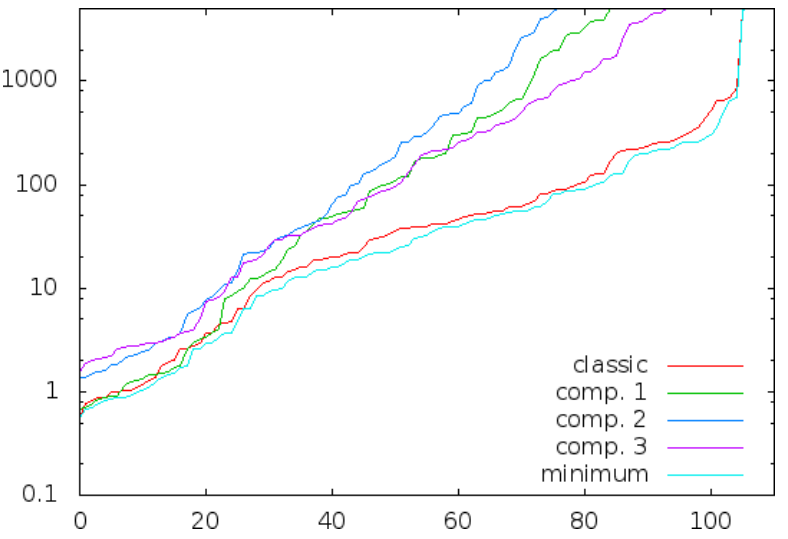

Figure 8: Performances for 108 AMBA benchmarks. The classical algorithm was able to solve 106 benchmarks, comp. 1 84, comp. 2 76, comp. 3 93.

\section{References}

[1] Henrik Reif Andersen (1997): An introduction to binary decision diagrams. Technical Report, Course Notes on the WWW. 
[2] Krzysztof R. Apt \& Erich Grädel (2011): Lectures in game theory for computer scientists. Cambridge University Press, doi $10.1017 / \mathrm{CBO} 9780511973468$.

[3] Roderick Bloem, Barbara Jobstmann, Nir Piterman, Amir Pnueli \& Yaniv Sa'ar (2012): Synthesis of Reactive(1) designs. Journal of Computer and System Sciences 78(3), pp. 911 - 938, doi:10.1016/j.jcss.2011.08.007. Available at http://www.sciencedirect.com/science/article/ pii/S0022000011000869. In Commemoration of Amir Pnueli.

[4] Romain Brenguier, Guillermo A. Pérez, Jean-François Raskin \& Ocan Sankur (2014): AbsSynthe: abstract synthesis from succinct safety specifications. In: Proceedings 3rd Workshop on Synthesis, SYNT 2014, Vienna, Austria, July 23-24, 2014., pp. 100-116, doi 10.4204/EPTCS.157.11.

[5] Randal E. Bryant (1986): Graph-based algorithms for boolean function manipulation. Computers, IEEE Transactions on 100(8), pp. 677-691, doi:10.1109/TC.1986.1676819.

[6] Jerry Burch, Edmund M Clarke \& David Long (1991): Symbolic model checking with partitioned transition relations. Computer Science Department, p. 435.

[7] Edmund M. Clarke, Orna Grumberg \& Doron Peled (2001): Model checking. MIT Press, doi:10.1016/B978044450813-3/50026-6.

[8] E. Allen Emerson \& Charanjit S. Jutla (1991): Tree automata, mu-calculus and determinacy. In: FOCS, IEEE, pp. 368-377, doi:10.1109/SFCS.1991.185392

[9] Emmanuel Filiot, Naiyong Jin \& Jean-François Raskin (2010): Compositional Algorithms for LTL Synthesis. In: Automated Technology for Verification and Analysis - 8th International Symposium, ATVA 2010, Singapore, September 21-24, 2010. Proceedings, Lecture Notes in Computer Science 6252, Springer, pp. 112-127, doi:10.1007/978-3-642-15643-4_10.

[10] Emmanuel Filiot, Naiyong Jin \& Jean-François Raskin (2011): Antichains and compositional algorithms for LTL synthesis. Formal Methods in System Design 39(3), pp. 261-296, doi:10.1007/s10703-011-0115-3.

[11] Guillermo A. Pérez: LTL2AIG. https://github.com/gaperez64/acacia_ltl2aig.

[12] Thomas R. Shiple, Ramin Hojati, Alberto L. Sangiovanni-Vincentelli \& Robert K. Brayton (1994): Heuristic minimization of BDDs using don't cares. In: Proceedings of the 31st annual Design Automation Conference, ACM, pp. 225-231, doi $10.1145 / 196244.196360$.

[13] Fabio Somenzi (1999): Binary Decision Diagrams. In: Calculational system design, 173, IOS Press, p. 303.

[14] Alfred Tarski et al. (1955): A lattice-theoretical fixpoint theorem and its applications. Pacific journal of Mathematics 5(2), pp. 285-309, doi:10.2140/pjm.1955.5.285.

[15] H.J. Touti, H. Savoj, B. Lin, R.K. Brayton \& A. Sangiovanni-Vincentelli (1990): Implicit enumeration of finite state machines using bdd's. In: IEEE Int. Conference on CAD, doi 10.1109/ICCAD.1990.129860.

[16] Chao Wang, Gary D Hachtel \& Fabio Somenzi (2003): The compositional far side of image computation. In: Proceedings of the 2003 IEEE/ACM international conference on Computer-aided design, IEEE Computer Society, p. 334, doi:10.1109/ICCAD.2003.159708. 